\title{
Germanica
}

\section{La cohabitation des âges dans le théâtre contemporain de langue allemande}

Accommodating different ages in contemporary German-language theatre

Das Zusammenleben der Lebensalter im zeitgenössischen deutschsprachigen

Theater

\section{Andreas Häcker}

\section{(2) OpenEdition}

\section{Journals}

Édition électronique

URL : http://journals.openedition.org/germanica/1627

DOI : 10.4000/germanica. 1627

ISSN : 2107-0784

Éditeur

Université de Lille

Édition imprimée

Date de publication : 10 juillet 2012

Pagination : 11-32

ISBN : 9782913857292

ISSN : 0984-2632

Référence électronique

Andreas Häcker, "La cohabitation des âges dans le théâtre contemporain de langue allemande », Germanica [En ligne], 50 | 2012, mis en ligne le 03 septembre 2012, consulté le 06 octobre 2020. URL http://journals.openedition.org/germanica/1627 ; DOI : https://doi.org/10.4000/germanica.1627

Ce document a été généré automatiquement le 6 octobre 2020.

(c) Tous droits réservés 


\title{
La cohabitation des âges dans le théâtre contemporain de langue allemande
}

\author{
Accommodating different ages in contemporary German-language theatre \\ Das Zusammenleben der Lebensalter im zeitgenössischen deutschsprachigen \\ Theater
}

Andreas Häcker

1 Le théâtre est un lieu pour regarder ce qui nous terrifie. La maladie et la mort, associées dans notre imaginaire au vieillissement, apeurent la scène. Condensant le temps et glorifiant le présent, elle peine à saisir la vieillesse et la fin de vie. À défaut de trouver une représentation adéquate, le théâtre enlaidit ou embellit les images de la vieillesse, comme la prose, en optant soit pour le dénigrement montrant les vieux comme avares, mesquins, hypochondriaques et misanthropes, soit pour l'éloge considérant la défaillance corporelle comme un espace de liberté pour l'esprit et la mémoire ${ }^{1}$. Face aux nouvelles formes de vie dans les sociétés qui vieillissent, il s'agit en particulier d'étudier le cadre dramaturgique que constitue la cohabitation scénique de plusieurs âges de vie. Enjeu riche, la cohabitation des personnages jeunes et vieux, cohabitation intime plus ou moins forcée, génère actions et affrontements. Pour parvenir à une vision globale de la diversité de ces cohabitations et des images de la vieillesse dans le théâtre contemporain germanophone, une première étape a consisté à réunir un corpus de travail. Comme les textes dramatiques contemporains sont plus facilement accessibles comme manuscrits chez auteurs, théatres ou éditeurs qu'en bibliothèque, nous avons mené, avec profit, un sondage auprès de plusieurs artistes, théâtres et maisons d'édition ${ }^{2}$. À partir de ce premier corpus réuni, nous avons prélevé un échantillon de quinze pièces abordant la vieillesse et les conflits intergénérationnels. Nous avons observé dans quelle mesure le décalage entre les expériences de vie, les attentes à l'égard de l'autre, l'envie de liberté et la crainte de dépendance perturbent les personnages. De nombreux textes, désignés comme comédies, ont suscité une interrogation complémentaire. Est-ce que ce sont uniquement les stéréotypes comiques 
et l'exagération burlesque qui permettent d'affronter au théatre les finitudes angoissantes que sont la vieillesse et la mort?

\section{L'heure du bilan : la retraite comme seuil d'entrée de la vieillesse}

2 La question est simple. Qui se dit délibérément vieux ? Dans un monde du paraître et du culte mercantile de la jeunesse éternelle ${ }^{3}$, il est difficile d'avouer son âge. Définir la vieillesse par un chiffre mathématique, en années de vie, est problématique. Dans une perspective biologique, nous vieillissons dès notre naissance de manière progressive, graduelle et très individuelle comme le notent Andreas Kruse et Hans-Werner Wahl, deux gérontologues: "Vieillir est un processus s'étendant sur toute la vie qui commence par la naissance et se termine par la mort», une définition claire de la vieillesse serait « impossible » bien que la période soit déterminée par des conventions sociales et des paramètres médicaux ${ }^{4}$. Aujourd'hui, la retraite de la vie professionnelle marque en particulier l'entrée dans la vieillesse. Se retirer de la vie active permet-il vraiment de définir le début du dernier cycle de vie? Sans charges de travail peut-on enfin entamer une vie épanouie? Philipp Löhle, auteur trentenaire, né en 1978, place ces réflexions au cœur de sa pièce Morgen ist auch noch ein Tag (On verra demain), créée au Theater Baden-Baden en 2009. La pièce montre les difficultés qu'éprouvent un "jeune » retraité et sa famille, elle saisit intuitivement les conflits qui se dessinent à l'horizon pour la société allemande affrontant une dynamique gériatrique ${ }^{5}$. Le programme distribué aux spectateurs suggère une mise en perspective inquiétante: « Notre société se retrouve dans un changement démographique. Nous vivons dans un monde où il y aura toujours davantage de personnes âgées. [...] Quelqu'un qui se retire du monde professionnel vers 50 ans aujourd'hui, peut avoir 100 ans sous certaines conditions. Un demi-siècle de temps libre ${ }^{6} »$. Les personnages principaux sont Karl et Katja Auer, un couple de sexagénaires dont les fils apparaissent pendant les communications téléphoniques. Des relations comptables se sont tissées entre les membres de cette famille issue des couches moyennes. L'auteur démarre la pièce par un count down biographique d'une génération qui ne traduira aucune satisfaction. Tandis que l'épouse maintient sa vie hyperactive dans le monde associatif, terrain qu'elle réserve à elle-même, le mari est confronté à un vide. Il souffre de l'inactivité soudaine qu'il n'a point préparée. Les comparaisons sont atroces. Katja souligne qu'elle restera, statistiquement, la plus jeune du couple. 


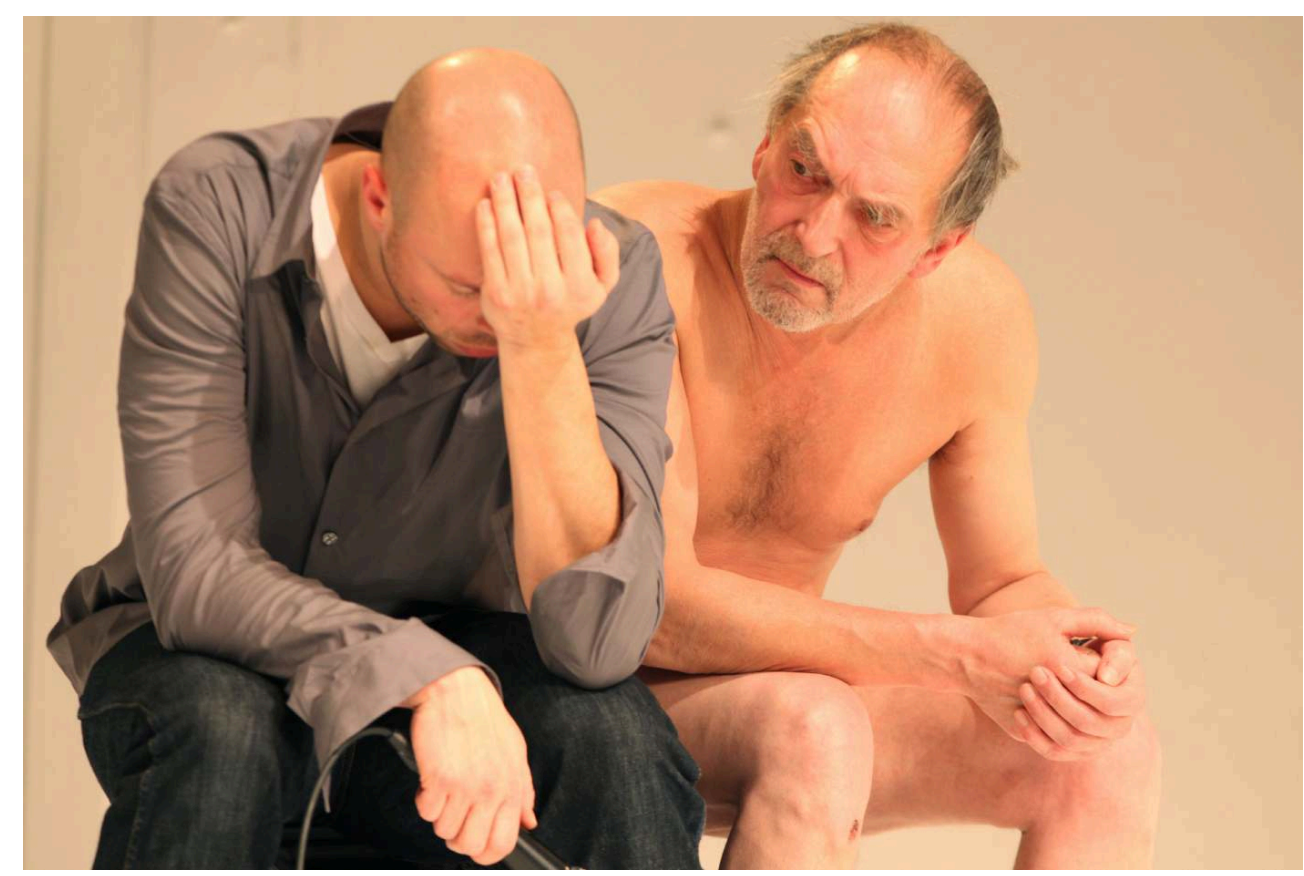

Nis-Momme Stockmann, DER MANN DER DIE WELT ASS,

Badisches Staatstheater Karlsruhe 2011

Daniel Stock et Ronald Funke jouent le fils et le père souffrant de démence.

Photo : Markus Kaesler

KATJA : En moyenne, les femmes vivent presque dix ans de plus que les hommes. C'est pourquoi, en regardant les choses par la fin, je suis plus jeune que toi. Quand tu as encore vingt ans à vivre, j'en aurai encore trente. Quand il t'en restera encore dix, j'en aurai toujours vingt, et quand tu seras mort, je devrai encore passer dix ans.

KARL: Mais je ne suis pas vieux à cause de ça. Pourquoi serais-je soudainement vieux? Juste parce que je ne travaille plus ? ${ }^{7}$

3 Morgen ist auch noch ein Tag est une pièce philosophique. Elle réfléchit sur notre incapacité de savourer la vie, sur les obsessions tyranniques du quotidien petitbourgeois, les schémas de la vie familiale. Si la vieillesse est synonyme de procrastination permanente, d'une vie d'oisiveté infinie, le diable, ce sera bien évidemment l'ennui. Abandonné par sa femme et ses fils, Karl Auer rencontre un groupuscule de vieux qui ont déclaré la guerre aux jeunes actifs. Ils terrorisent les jeunes en provoquant des accidents de voiture. Selon ces vieux vampiriques accrochés à leurs déambulateurs, la jeunesse doit payer pour le grand âge. La terroriste Lore affirme :

C'est tout simple. Tu te mets au bord d'une grande route ou d'un passage piéton - le meilleur moment c'est entre sept et neuf heures du matin et cinq et sept heures du soir - tu attends jusqu'à ce qu'une voiture passe. S'il est encore à dix, quinze mètres de distance, tu sautes sur la route ou sur le passage piéton, devant sa voiture. Dans le meilleur des cas, tu te casses un bras ou tu te tords la cheville. [...] Le jeunot perd son permis et son assurance complète ta retraite ${ }^{8}$.

Notamment les jeunes dramaturges abordent la difficulté d'assumer le vieillissement des membres de la famille et la solidarité entre les générations. Dans sa pièce Der Mann der die Welt aß (L'homme qui a mangé le monde) ${ }^{9}$, lauréat du Stückemarkt Heidelberg en 2009, Nis-Momme Stockmann (né en 1981) s'interroge sur les rapports entre un fils trentenaire arrogant qui est au chômage et son père, veuf sexagénaire souffrant de démence. Avec une précision réaliste, Stockmann dessine deux hommes qui entament 
une cohabitation délicate. À travers plusieurs coups de téléphone, il décrit, au début de la pièce, les premiers symptômes de démence qui se manifestent chez le père. Lisa, l'exfemme du fils, appelle ce dernier et raconte les multiples appels de son père. Le fils semble ne pas accepter la sénilité de son père qui nécessite une prise en charge.

LISA : Sans ta mère, il ne va pas bien. [...] Il a raconté aux enfants l'histoire de sa langue [qu'il a mordue]. Et celle de ses ampoules sur sa main brûlée [...] Il ne va pas bien.

LE FILS (soupire) : Oui, il est enrhumé.

LISA : Je crois... mais vraiment tu dois y penser... Je crois qu'il a besoin de quelqu'un. LE FILS : Foutaise. Ce n'est pas si grave.

LISA : Il devient vieux. Et confus dans la tête. Il ne se débrouillera plus tout seul.

LE FILS : Il a seulement une petite grippe.

LISA : Et pourquoi se mord-il la langue ? Il a de si mauvaises dents. [...] Il a besoin

d'être pris en charge.

LE FILS : Il a seulement 64 ans.

LISA : 67 ans.

LE FILS : Mais il continue de travailler.

LISA : Oui, c'est justement pour ça. S'il fait juste une erreur... Il n'est même pas assuré, pour rien ${ }^{10}$.

5 Par son écriture intime, Der Mann der die Welt a $\beta$ choisit une voie un peu exceptionnelle. Souvent les pièces de théâtre passent par la caricature et l'image grotesque pour décrire des personnes âgées et leur état d'affaiblissement.

\section{Le vieillissement comme effraction de la laideur}

6 La littérature théâtrale associe fréquemment la vieillesse aux images effrayantes de la maladie, de la sclérose, du délabrement physique et moral. Thomas Bernhard (1931-1989) excelle dans la peinture des fêtes réunissant retraités aliénés et vieillards sadiques. Ein Fest für Boris (Une fête pour Boris) se termine par la mort du protagoniste assis dans un fauteuil roulant, une image qui rappelle le théâtre de Samuel Beckett ${ }^{11}$. Dans Minetti de Bernhard ${ }^{12}$, le personnage principal est un vieil artiste. Ancien acteur qui vit en retrait du théâtre depuis plusieurs décennies, il se rend à Ostende pour attendre un directeur de théâtre qui lui aurait proposé de jouer le rôle de Lear, roi sombrant dans la folie, incapable de transmettre son héritage. Assis à côté d'une jeune fille de dix-sept ans, Minetti fait le bilan de sa vie en suivant des yeux les hôtes de l'hôtel, un nain, un vieux couple, des fous masqués d'une nuit de la Saint-Sylvestre. Avant de se suicider dans une tempête de neige, il sort de sa valise, fardeau du nomade échoué, le masque de Lear créé par Ensor. D'autres auteurs rapprochent également vieillesse, folie et nostalgie mélancolique. Comme dans le théâtre de Tennessee Williams ${ }^{13}$ ou de Tchekhov où les figures ont du mal à accepter de se voir faner, les personnages ne supportent guère de se regarder chez Wilhelm Genazino, auteur né en 1943 et lauréat du prix Georg Büchner en 2004. Lieber Gott mach mich blind, (Miséricorde, rends-moi aveugle) s'ouvre par une discussion entre l'épouse et l'amante d'un homme sexagénaire. Martha, l'épouse svelte de 60 ans, exprime son dégoût à l'égard de Robert, son mari obèse. Observatrice impitoyable, elle compare ses sacs lacrymaux aux petits sacs d'aspirateur. L'amante l'interpelle :

IRIS : Vous ne parlez plus de vos visages?

MARTHA : Nous ne voulons plus revoir nos visages. [...] Je comprends chacun qui m'a connu il y a vingt ans et qui ne veut plus me regarder. [...] Dans ma jeunesse, j'ai été 
blanche comme la porcelaine et douce comme un ange. Robert m'a regardée et il a pensé : Mon Dieu, elle ressemble à un verre de lait. [...] Je me suis senti comme un verre de lait. À un moment donné, quelqu'un saisit mes lèvres et m'avale. À ce moment-là, je suis sauvée ${ }^{14}$.

7 La haine et le mépris infiltrent les relations. Avancer en âge, c'est avancer dans le dégoût des autres. On ne peut plus croire dans les contes de fées évoquant les forces de régénération magique. Signe de fécondité et de bonne santé chez les princesses, la chevelure exprime l'altération négative du corps et la perte des forces de séduction amoureuse chez la vieille femme.

MARTHA : C'est inimaginable à quel point j'étais satisfaite de mes cheveux jadis! Quand j'avais neuf ans, je regardais dans le miroir et immédiatement j'étais heureuse. Sans cesse, presque, je me peignais. Enfant, je perdais déjà des cheveux. Mais à cette époque, la chute des cheveux a été quelque chose de merveilleux. À cette époque, mes cheveux repoussaient rapidement. [...] Maintenant, mon cuir chevelu transparaît de plus en plus. Je suis dégoûtée par la couleur rose de ma tête qui devient chauve. La couleur rose chez des femmes d'un certain âge veut dire : faites attention, je vais vous dégoûter! Hélas, j'ai mis tant d'espoirs dans mes cheveux ${ }^{15}$.

8 Tous les personnages sont assaillis par le vieillissement. La belle-fille trentenaire affronte déjà les signes ravageurs qui surgissent sur son visage. Iris, amante miroir, interroge Robert avec insistance pour connaître les véritables raisons de la répulsion qu'il éprouve. Le désespoir est au rendez-vous quand il mesure l'amour uniquement comme une performance physique.

ROBERT : [...] Tiens, quand nous nous aimions, nous étions encore jeunes. Il a suffi de nous coucher l'un à côté de l'autre, il a suffi de nous asseoir à une table. Il y a longtemps. Aujourd'hui, nous pouvons rester couchés l'un à côté de l'autre, rien ne bouge, ni chez toi ni chez moi. [...] Quand deux personnes ont vieilli et que l'amour ne réussit plus, il y a deux possibilités. La première : le couple est sensé, estime la fatigue comme naturelle et arrête l'affaire. La deuxième : le couple s'invente une perversion, permettant de perpétuer la sexualité, par d'autres moyens pour le dire ainsi ${ }^{16}$.

Genazino aborde la déchéance également dans sa pièce Der Hausschrat (Le Génie de la maison), dont le titre est un clin d'œil à la pièce tchekhovienne Le Génie de la forêt. Le personnage central est Karl, un homme quinquagénaire, cynique et casanier. Karl commence à flirter avec Else, quadragénaire et compagne de son frère qui vient de mourir. Tandis que Sophie, la femme de Karl, se rend à l'enterrement, Karl souhaite échanger Sophie contre Else qui lui pose des questions précises sur une vie conjugale potentielle. Elle interroge Karl sur son hygiène de vie, sur ses troubles du sommeil. Et bien plus.

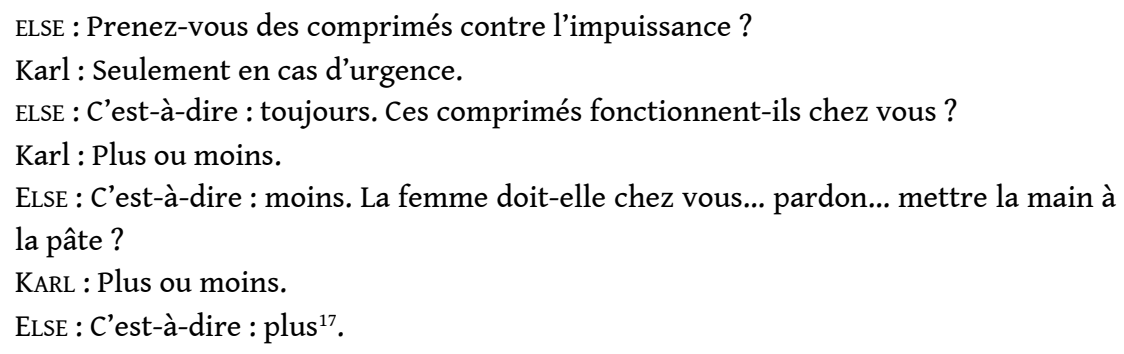

Les auteurs contemporains ne s'arrêtent pas à l'impuissance sexuelle ou à la lutte contre l'ennui et la solitude. Le discours médical envahit l'écriture dramatique. Un accident vasculaire bouleverse la vie rangée d'un couple de seniors dans Liebesruh 
(Repos d'amour), une pièce de Jan Neumann créée en octobre 2005 au Thalia Theater Hamburg. L'auteur, né en 1975, montre, en une trentaine de scènes, la vie de Regine et Karl, un couple proche de la retraite. Pendant un séjour de vacances à l'étranger, la femme est frappée d'une attaque d'apoplexie. Hospitalisée en Allemagne, Karl se rend tous les jours au chevet de son épouse. Elle reste paralysée et ne peut communiquer que par les mouvements des yeux. À travers les dialogues avec les médecins, on apprend la dégradation perpétuelle de son état et la dépression qui s'installe chez Karl. Son frère tend vainement de le persuader de trouver pour sa femme une maison de retraite médicalisée. La sobriété du dialogue est touchante, elle saisit de manière précise la faiblesse et le dilemme dans lequel se trouvent les personnes qui doivent placer un parent malade ou dépendant.

Karl : Elle fait des progrès.

Frère : Elle est paralysée. Elle ne peut pas parler.

Karl : Je peux m'occuper d'elle.

FRÈre : Tu n'es plus tout jeune.

Karl : J'y arriverai.

FRÈRE : C'est trop pour toi.

Karl : Pas de problème.

FRÈRE : C'est égoïste.

Karl : C'est toi qui le dis.

FRÈRE : Et si tu cherchais une maison de retraite?

Karl : Non.

FRÈRE : Pourquoi pas?

Karl : Elle ne veut pas aller dans une maison de retraite ${ }^{18}$.

11 Refusant catégoriquement le placement de sa femme, Karl donne des somnifères à son épouse vers la fin de la pièce. La dramaturgie joue sur les ambiguïtés de cette situation. Est-ce que Karl assassine Regine contre son gré ou est-ce qu'il l'assiste dans un suicide sur demande?

Karl : On veut te transférer dans un établissement médicalisé.

REGINE : Non.

Karl : J'ai des comprimés.

Karl : Tu t'endormiras et tu ne te réveilleras plus.

REGINE : Oui. [...]

Elle s'endort.

Karl : J'ai peur.

Il l'étrangle ${ }^{19}$.

12 Les figures de la fin de vie sont assez ténébreuses dans le théâtre germanophone contemporain qui dessine souvent des vieillards sombrant dans la débilité. La pièce néopopulaire de Ralf-G. Krolkiewicz (1955-2008), dont le ton ressemble au ton cru chez Werner Schwab, sonst is alles wie immer (comme d'habitude) montre une famille en pleine décomposition. Avec enfants et amants, agressifs et alcooliques, quatre filles se réunissent pour fêter le quatre-vingt-dixième anniversaire d'Ede, leur père incestueux. Placé en foyer, il est ramené par deux filles à la maison familiale. Lene, une de ses quatre filles, évoque l'état de santé du père qui ressemble à un légume putréfié :

est-ce qu'il remarque encore la différence / entre ces médocs / et disons / un sandwich au pâté / il bouffe l'un comme l'autre / sans réfléchir / et sans appétit / seulement parce que quelqu'un lui dit / bouffe maintenant Ede / et il l'avale / franchement / veux-tu que ta vie / soit prolongée ainsi / dans cet état / par violence dans la pénombre / entre vie et mort / être remis de force sans cesse / tandis que la mort serait soulagement ${ }^{20}$. 
13 Chez Theresia Walser, née en 1967, abondent les images burlesques, sombres et scatologiques. Dans King Kongs Töchter (Les Filles de King Kong), pièce créée en 1998 au Theater am Neumarkt à Zurich, les pensionnaires d'une maison de retraite médicalisée souffrent de démence, diabète, troubles psychiatriques et incontinence. Les trois aidessoignantes Meggie, Berta et Carla sont bavardes, mais cachent prudemment leur profession face à leurs amants potentiels.

BERTA: Quand je fais connaissance d'un homme, je ne lui parle jamais de ma profession. [...] Le plus souvent, je dis que je m'appelle Marie et que j'ai un salon de manucure.

CARLA : En tout cas, ça sonne mieux que: «Je m'appelle Berta, l'aide-soignante gériatrique ».

BERTA Ou : «Je suis Carla, la dompteuse de seniors ».

CARLA Ou : «Berta qui torche les vieillards $»^{21}$.

14 Ces trois femmes ont une obsession. Elles jouent aux anges de la mort embellissant les derniers instants des agonisants comme dans un film hollywoodien ${ }^{22}$. Prolongeant le culte des momies, elles placent les pensionnaires sur des canapés et célèbrent leur mort comme un événement esthétique.

MEGGIE : Et quand quelque chose crève ici, quelque chose qui ressemble à Ötzi, s'il le faut, j'y repasserai après le fer à souder; quand on la regarde, il faut croire, au moins, qu'Ötzi aurait niqué encore la Sophia Loren. [...] Et oui, oui, il faut exagérer, autrefois, on leur a tissé des raisins dans les cheveux, rempli leur bouche de blé et ... $\mathrm{Ciao}^{23}$.

Dans la cinquième scène de la pièce, les hôtesses de la mort transforment une octogénaire en Mae West, femme fatale, vedette voluptueuse et licencieuse du cinéma des années 1930.

BERTA : Doucement, ma vieille fille, doucement. Cette nuit, je ferai de toi le grand kitsch, la déesse du sexe sorti des fonds de placard, dans ta chute, je repose tout mon cœur.

MEGGIE : Ne lui dessine pas une bouche trop grande, Berta.

BERTA : Avoir 80 ans, eh bien ma vieille petite souris, avoir 80 ans [...] mais qui veut regarder comment tu t'enfonces lentement dans le matelas ici $?^{24}$

Bien vieillir et mourir en beauté, est-ce vraiment possible? L'écriture dramatique enjolive les défaillances communes avec humour, les maisons de retraite sont des cages aux fous.

\section{Embellir l'image de la vieillesse : vers une nouvelle solidarité intergénérationnelle}

17 Dans Gesegnete Mahlzeit (Bon appétit) ${ }^{25}$, Karl Wittlinger (1922-1994) décrit une pension de famille pour aînés gérée par un couple avide qui profite de la situation précaire des pensionnaires. Elfie, personnage principal de la comédie, vient de sortir de prison et s'installe dans la pension où une place vient de se libérer. N'acceptant pas les rituels humiliants et réclamant davantage d'autonomie, Elfie remonte les pensionnaires contre les gérants tyranniques. Nourrie de l'esprit rebelle des vieux animaux dans Die Bremer Stadtmusikanten, conte des frères Grimm, elle affirme que la vieillesse ne rime pas avec abnégation, austérité et ascèse. Elle invite ses voisins à puiser dans les joies de la vie. Au lieu d'avaler des tisanes fades et grignoter des sablés secs, il faut lubrifier la machinerie rouillée - à l'aide de forts stimulants comme magazines érotiques et alcool. 
ELFIE (les interrompt) : Et bien -: Quand les années arrivent où nous, les filles, n'obtenons plus ce dont nous avons besoin, et les garçons ne peuvent plus vraiment faire ce qu'ils veulent faire, s'installe, comme on dit, "la frustration de la vieillesse ». En cause : les hormones, comme un célèbre chercheur l'a pigé dans son laboratoire. Et il a conclu que le porno en est un remède appropriée ${ }^{26}$.

s'agit de saisir sa place, de ne pas souffrir d'une dépendance autant choisie que subie. Solution, encore insolite pour les années 1990, les pensionnaires dans cette comédie songent à fonder une colocation de seniors pour gérer différemment la vieillesse. Markus Köbeli, auteur suisse né en 1956, est déjà plus progressif dans sa comédie Zimmer frei (Chambre à louer) ${ }^{27}$. Il crée une colocation intergénérationnelle qui donnera lieu à un apprentissage mutuel. Glotz, une jeune femme gothique, cherche un colocataire. Grand-père, ancien fonctionnaire du ministère des finances, expulsé de sa maison, attend qu'une place se libère dans une résidence pour personnes âgées. Il vient visiter l'appartement. La mort est présente dès le début. Glotz se lève de son lit qui est un cercueil.

(Silence. Grand-père regarde en direction du cercueil.)

GLOTZ : Ma dernière acquisition. Chic, n'est-ce pas?

GRAND-PÈRE : Très élégant, oui.

GLOTZ : Subtil Élégant? Il est complètement destroy! Vous savez combien coûte un machin comme ça ? Il faut en mettre du pognon.

GRAND-PÈRE : Je sais. Je me suis déjà renseigné.

GLOTZ : Pourquoi ? Vous craquez aussi pour les cercueils?

GRAND-PÈRE : Non, non. Mon intérêt est tout simplement lié à mon âge. J'aime l'ordre dans les affaires, vous comprenez?

GLOTZ: Je préfère plutôt le chaos. Vivre, mais tout de suite! C'est pourquoi j'ai acheté ce cercueil. ${ }^{28}$

Dans la pièce de Köbeli, deux modes de vie s'entrechoquent avec beaucoup de tendresse. Rappelant éros et thanatos, Glotz et Grand-Père sont unis dans l'utopie d'une vie heureuse. Au lieu d'attendre la mort dans la maison de retraite, le vieil homme reste colocataire et vivra les moments béats qu'il aurait voulu vivre avec son épouse comme une escapade en moto sur les routes de Normandie, le paradis rêvé. Grand-père mourra dans les bras de Glotz qui devient son âme sœur.

La peur de perdre l'autonomie stucture la pièce Das Herz eines Boxers (Le Cour d'un boxeur), créée au Grips Theater de Berlin en 1996. Lutz Hübner, né en 1964, nous montre la relation chaleureuse entre Léo, ancienne légende de la boxe berlinoise, hospitalisé dans un service de psychiatrie fermé, et Jojo, un garçon de 17 ans qui accomplit des travaux d'intérêt général après un vol de mobylette. Il doit repeindre la chambre de Léo qui refuse les soins.

Léo entre, sur la table une serviette propre et un petit récipient avec des comprimés. Léo met la serviette sur l'épaule, verse les comprimés dans le creux de sa main, va vers la fenêtre, il l'ouvre, regarde autour de lui et jette les comprimés en faisant un grand mouvement, il ferme la fenêtre ${ }^{29}$.

Dans la première scène, Léo reste muet et subit l'entrée fracassante de Jojo qui déballe une panoplie de remarques stéréotypées à l'égard des personnes âgées et leur sénilité présumée. Jojo dit :

Les pantoufles et le gilet tricoté restent propres, on t'emballe tout de suite joliment dans un film, j'ai apporté même du film transparent pour que tu puisses, fort impressionné, me regarder peindre les murs. C'est vraiment rare que quelqu'un bosse ici à la maison de retraite, n'est-ce pas ? Bien sûr, des étoiles en paille et les autres trucs, un vrai travail de cochon. [...] Pourquoi tu me fixes comme ça? La 
première visite depuis la guerre, n'est-ce pas? Ne crois surtout pas que je fais ceci par philanthropie. Ai-je l'air d'un bienfaiteur des seniors ? ${ }^{30}$

Jojo et Léo s'apprivoisent au fur et à mesure des visites de Jojo. Léo revient sur son enfermement dans le service psychiatrique. Se rebellant contre les traitements humiliants, il a frappé un aide-soignant qui lui infligeait un langage infantilisant ${ }^{31}$. Léo raconte :

Avant, j'étais dans une maison de retraite où je me sentais vraiment bien. [...] une fois, j'ai mouillé mon pantalon, j'ai eu très honte. Tu sais, ce n'est pas toujours simple d'être vieux quand les gens te traitent comme un idiot. Et il y a eu cet aidesoignant, je n'ai jamais pu le supporter, un mec comme toi, comme tu es entré ici avant-hier, un mec qui te traite comme un cochon d'Inde. Il vient chercher le linge et remarque ma mésaventure. Il gueule pour que tout le monde puisse l'entendre : Hé, Léo, je crois que c'est le moment de te mettre des couches. J'ai eu très honte, mais j'ai pensé : Tu es seulement un petit morveux, tu deviendras vieux, toi aussi, plus vite que tu le penses. Mais quand il revient le lendemain et me demande avec un sourire mesquin si j'étais encore bien sec, j'ai eu un coup de colère. Tu sais, autrefois, j'ai été célèbre pour mon gauche, ils m'appelaient le marteau d'acier. À l'instant, il est tombé par terre [...] $»^{32}$

Léo apprend au jeune homme à canaliser sa violence et sa haine. Grâce aux conseils du boxeur, connaisseur des recettes d'amour infaillibles, Jojo réussit à charmer une fille qu'il n'osait pas aborder depuis longtemps. La relation entre le jeune homme et le vieil homme est basée sur la solidarité, le respect de l'autre et de son désir de vivre en autonomie. À la fin, Jojo aide Léo à s'échapper du service psychiatrique fermé, il peut rejoindre un ancien ami boxeur, aveugle, qui tient un bar dans le sud de la France, symbole de la liberté3 ${ }^{33}$.

Peter Turrini, auteur autrichien né en 1944, accorde une place importante au jeu de deux comédiens âgés dans sa pièce Josef und Maria (Joseph et Marie). Souhaitant écrire une pièce comportant une conclusion heureuse $\mathrm{e}^{34}$, Turrini retrace son approche humble à l'égard des personnes âgées et de leurs souvenirs :

"L'image que possède la société des personnes âgées est peu déterminée par les vieux eux-mêmes. La publicité les découvre comme une "strate spécifique d'acheteurs", la télévision fait d'eux des "seniors" joyeux, et même la sociologie scientifique valide ses propres prémisses chez les personnes âgées : elles sont une minorité défavorisée. Je n'ai pas voulu écrire une pièce "sur" les personnes âgées. J'ai voulu enregistrer et accepter leurs histoires, leurs récits, leurs souvenirs. Ma tâche comme auteur dramatique a consisté à choisir ces histoires, à les mettre dans un bon ordre et à introduire deux personnes âgées dans une situation dramatique » 35 .

L'auteur nous montre une vieille femme et un vieil homme qui passeront ensemble un réveillon de Noël dans un grand magasin. Maria fait le ménage, Josef est gardien. Renfermés dans une sorte d'autisme au début de la pièce, les deux personnages décrivent leur vie solitaire et partagent leurs lectures. Josef, révolutionnaire et vendeur d'un journal communiste, lit un poème d'un camarade résistant emprisonné par les nazis. Maria revient sur ses lectures de la presse féminine qui reflètent sa misère.

MARIE lit. «Je veux ouvrir mon cœur, en effet, personne ne pourra m'aider. Devant moi, ma belle-fille enferme à clé les vivres, je ne dois rien prendre et j'ai toujours faim. Mes amis me paient de temps en temps un café. Ma belle-fille encaisse ma pension, elle n'en lâche rien. J'ai 87 ans. Ai-je mérité cela pour mes vieux jours? Salutations distinguées »" Noël par sa belle-fille, une poissonnière avaricieuse qui mène une vie terne de petite- 
bourgeoise. Face à cette souffrance, Maria fait le bilan de sa vie, comptant les moments de bonheur qu'elle a vécus comme artiste de variété pendant sa jeunesse. Ces instants valent plus que quarante années passées à une chaîne de montage. Une heure de gloire efface une vie malheureuse détruite par le mépris des autres ${ }^{37}$. Josef possède aussi un passé d'artiste, mais son esprit rebelle a heurté l'esprit réactionnaire manifeste dans l'Autriche d'après-guerre et notamment au Burgtheater. Maria, qui souligne qu'elle a été une belle femme, cherche à séduire Josef. Le jeu de séduction échoue dans un premier temps, mais ensuite les protagonistes passent progressivement à l'acte charnel. Avec tendresse et humanité, ils font preuve de compréhension pour les faiblesses et les blessures de l'autre.

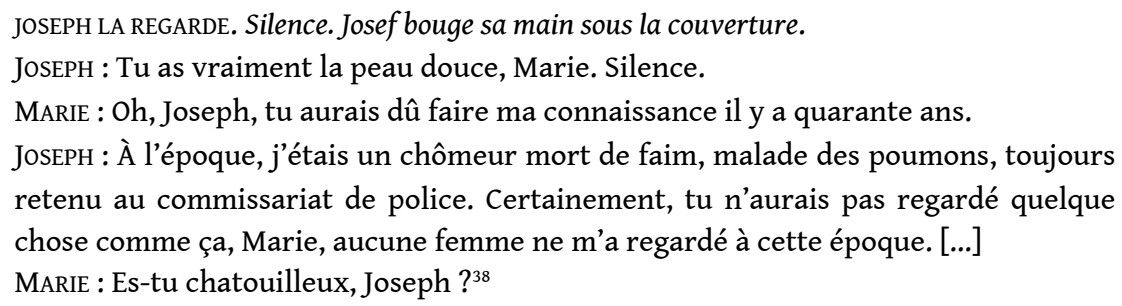

En toute décence, la pièce de Turrini se termine dans un rire joyeux. La vieillesse rend heureux et apporte le grand amour.

\section{Explorer les possibilités du changement}

L'étude du corpus initial a montré que la littérature théâtrale germanophone se rebelle souvent contre le vieillissement et choisit la voie de la laideur pour représenter le dernier cycle de vie. Mais à côté des pièces caricaturalement pessimistes, voire nihilistes, dans lesquelles règnent résignation et rancune, bon nombre d'artistes affirment que les personnes âgées peuvent commencer une nouvelle vie malgré la défaillance corporelle et apporter des richesses aux autres générations. Le théâtre amateur des seniors et l'écriture par le plateau proposent des visions confiantes, ils valorisent les témoignages des personnes âgées en poursuivant la conception esthétique que Peter Turrini a esquissée dans sa note d'intention concernant sa pièce Josef und $\mathrm{Maria}^{39}$ : pour eux aussi il s'agit d'accepter, de rassembler, de manière attentive et bienveillante, les histoires des vieux, leurs récits et leurs souvenirs.

Quand les souvenirs et la vie ordinaire s'exposent, la scène devient un lieu d'avenir. Katharina Kofmehl-Heri souligne "l'optimisme inébranlable $»^{40}$ du théâtre des seniors en Suisse, théatre populaire créé par les personnes âgées, souvent exclusivement pour un public du même âge, comme elle note en conclusion de sa thèse en ethnologie qui retrace l'histoire et la pratique de cette forme théâtrale qui s'est développée en Suisse alémanique et romande depuis les années 1970. Investissant les biographies des acteurs et les vécus générationnels, les pièces impliquent mémoires et aspects de la vie quotidienne. Elles abordent, par exemple, la difficulté d'accepter les prothèses auditives, le diabète ou les désirs amoureux cachés. L'ethnologue remarque que le jeu théâtral permet, particulièrement aux retraitées suisses, d'acquérir un espace de liberté et une reconnaissance sociale dans une société paternaliste qui maintient, même dans la vieillesse, le partage sexiste entre tâches ménagères et tâches publiques ${ }^{41}$. 


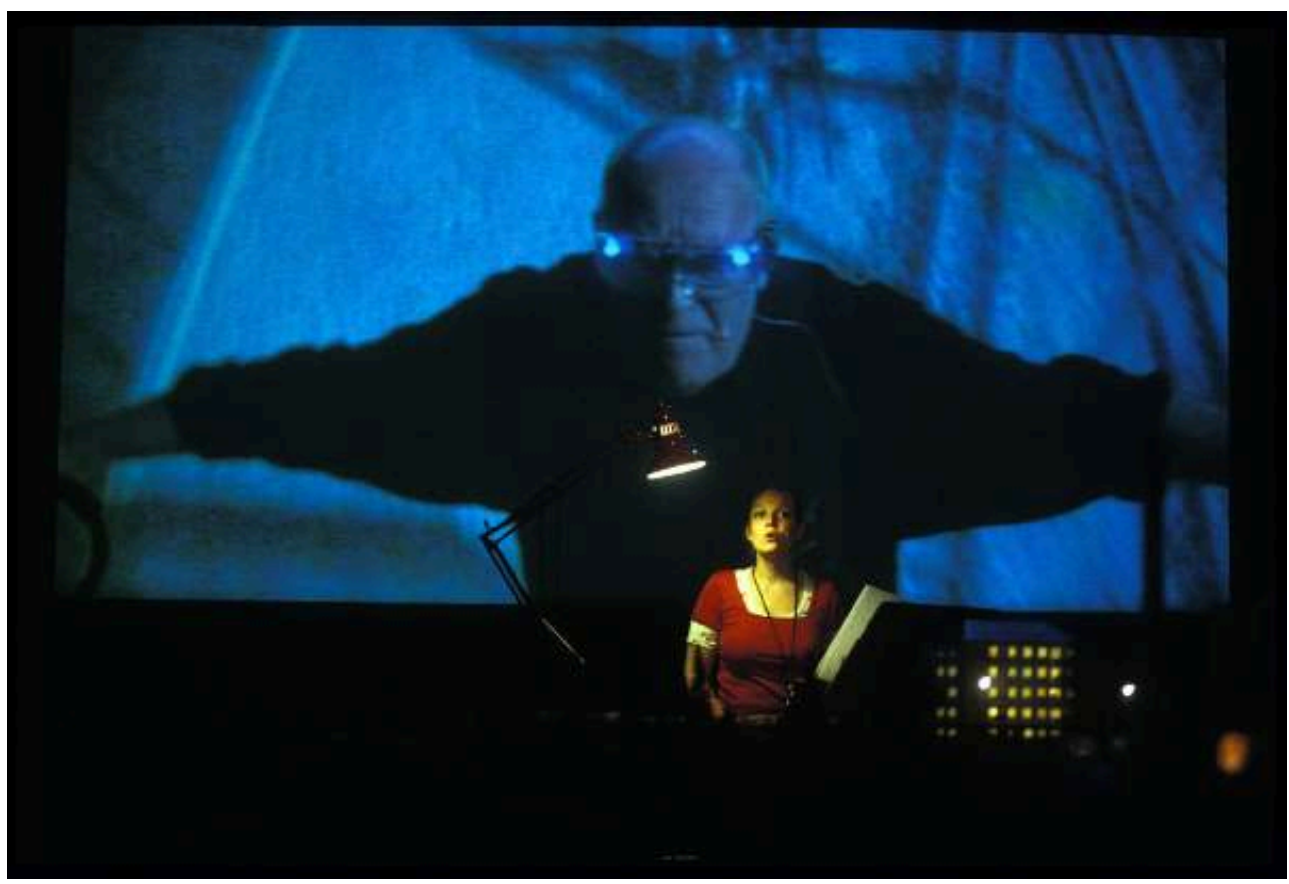

Acteurs : Max Kurrus, Rahel Hubacher. Photo : Sebastian Hoppe.

Les nouveaux créateurs de théâtre comme Helgard Haug, Stefan Kaegi et Daniel Wetzel sondent aussi les biographies des retraités. Leur label théâtral Rimini Protokoll a développé plusieurs projets documentaires comme Kreuzworträtsel Boxenstopp (Motscroisés arrêt au stand) ou Mnemopark ${ }^{42}$ où des personnes âgées racontent leurs souvenirs et leur difficulté de vieillir. Stefan Kaegi a réuni quatre modélistes âgés et une jeune actrice dans Mnemopark. Le spectacle, créé au Theater Basel en 2005, a présenté une Suisse en miniature observée par un œil critique. Kreuzworträtsel Boxenstopp, créé au Künstlerhaus Mousonturm en novembre 2000, a été porté par plusieurs femmes pensionnaires d'une maison de retraite à Francfort-sur-le-Main. Rimini Protokoll a juxtaposé leurs récits sur la vie au foyer et les réminiscences issues du monde automobile. Les isotopies de l'accélération et du temps mesuré sont au cœur de cette installation théâtralisée : des images filmées dans la maison de retraite se mêlent aux voitures de courses. La narratrice lit les récits de deux autres femmes âgées participant au projet :

Madame Döring avec le lance-pierre. Elle dit : Jadis, je pensais que c'est plus tard qu'on devenait vieux. Madame Simon dit que la vitesse maximale c'est quand je tombe et me casse le col de fémur. Vous ne pouvez pas représenter cette chute ${ }^{43}$.

31 Bien d'autres spectacles germanophones de la première décennie du xxI siècle affichent une forte implication biographique voire autobiographique. Le metteur en scène Sebastian Nübling rassemble les membres de sa famille dans Mütter.Väter.Kinder (Mères.Pères.Enfants) ${ }^{44}$, un spectacle de danse créé au Theater Freiburg en 2008 où il montre la vie commune de trois générations qui comparent, comme dans un jeu d'enfants, leurs compétences et leurs performances. Testament: Verspätete Vorbereitungen zum Generationenwechsel nach Lear (Testament: préparations tardives au changement générationnel d'après Lear) a été créé au théâtre Hebbel am Ufer à Berlin en 2010 par les artistes du collectif théâtral She She Pop qui explorent les conflits 
fondamentaux de la pièce shakespearienne comme la transmission de l'héritage aux enfants ingrats et les jalousies entre les frères et sœurs. Désirant dénuder les antagonismes qui ont généralement tendance à rester inavoués et tacites entre les membres d'une famille, Testament réunit les artistes de scène et leurs pères sur le plateau. Devant les spectateurs, on énonce les questions de succession patrimoniale, on discute les plans d'architecte pour installer un parent dépendant dans l'appartement des enfants. D'une manière mathématique, un acteur s'interroge même sur la valeur monétaire d'un grand-père pour calculer son "héritage réel » ${ }^{45}$. Cette récente objectivité sur les scènes germanophones confirme que la cohabitation des âges reste une affaire à négocier en permanence au théâtre comme dans la vie.

\section{CEuvres}

\section{BIBLIOGRAPHIE}

Thomas Bernhard, Minetti : ein Porträt des Künstlers als alter Mann, Suhrkamp, Frankfurt am Main 1977, création au Württembergisches Staatstheater Stuttgart, $1^{\mathrm{er}}$ septembre 1976.

Wilhelm Genazino, Lieber Gott, mach mich blind / Der Hausschrat, Hanser, München 2006, création Lieber Gott, mach mich blind au Staatstheater Darmstadt, 8 octobre 2005. création Der Hausschrat au Theater an der Ruhr, Mülheim, 13 février 2007.

Lutz Hübner, Das Herz eines Boxers, Klett, Stuttgart 2009 [Hartmann \& Stauffacher, Köln 1996], création au GRIPS Theater, Berlin, 19 octobre 1996.

Markus Köbeli, Zimmer frei, Gustav Kiepenheuer Verlag, Berlin 1990, création au Stadttheater Konstanz, 2 février 1991.

Ralf-G. Krolkiewicz, sonst is alles wie immer, Drei Masken Verlag, München 2003, création au Theater 89, Berlin, 2 février 2006.

Philipp Löhle, Morgen ist auch noch ein Tag, Verlag Autorenagentur, Berlin 2008, création au Theater Baden-Baden, 23 janvier 2009.

Jan Neumann, Liebesruh, Gustav Kiepenheuer Verlag, Berlin 2005, création au Thalia Theater Hamburg, 12 octobre 2005.

Sebastian Nübling/physical virus collective tanz heidelberg/freiburg, Mütter.Väter.Kinder, création au Theater Freiburg, 27 novembre 2008.

Rimini Protokoll, Kreuzworträtsel Boxenstopp, création au Künstlerhaus Mousonturm, Festival plateaux, Frankfurt am Main, 10 novembre 2000.

Rimini Protokoll, Mnemopark, création au Theater Basel, 24 mai 2005.

She She Pop \& ihre Väter, Testament : Verspätete Vorbereitungen zum Generationenwechsel nach Lear, création au Hebbel am Ufer/HAU 2, Berlin, 25 février 2010. 
Nis-Momme Stockmann, Der Mann der die Welt a $\beta$, schaefersphilippen, Köln 2009, création au Theater der Stadt Heidelberg, 17 décembre 2009.

Peter Turrini, Josef und Maria, Suhrkamp, Frankfurt am Main 2003 [Frischfleisch \& Löwenmaul, Wien 1980], création au Volkstheater Wien, ÖGB-Haus, 12 novembre 1980.

Theresia Walser, King Kongs Töchter, Verlag der Autoren, Frankfurt am Main 1999, création au Theater Neumarkt, Zürich, 26 septembre 1998.

Karl Wittlinger, Gesegnete Mahlzeit, Verlag Felix Bloch Erben, Berlin [1991], création au Ohnsorg Theater Hamburg, sous le titre Pension Sünneschien, 31 mars 1991.

\section{Critique et documentation}

Anne Deshoulières, "Le théâtre du “viellir" dans La ménagerie de verre et Un tramway nommé désir de Tennessee Williams », in Alain Montandon (éd.), Figures du vieillir, Presses Universitaires Blaise Pascal, Clermont-Ferrand 2005, pp. 173-193.

Franz Gebesmair et Manfred Mittermayer (ed.), Bernhard-Tage Ohlsdorf 1999. „In entgegengesetzter Richtung“: Thomas Bernhard und sein Großvater Johannes Freumbichler, Bibliothek der Provinz, Weitra 2000.

Andreas Kruse (éd.), Leben im Alter. Eigen- und Mitverantwortlichkeit in Gesellschaft, Kultur und Politik, AKA Verlag, Heidelberg 2010.

Andreas Kruse et Hans-Werner Wahl (éd.), Zukunft Altern. Individuelle und gesellschaftliche Weichenstellungen, Spektrum Wissenschaftlicher Verlag, Heidelberg 2010.

Helmuth Kiesel, « Das Alter in der Literatur », in Ursula M. Staudinger (éd.), Was ist Alter(n) ? Neue Antworten auf eine scheinbar einfache Frage, Springer, Heidelberg 2008, pp. 173-188.

Helmuth Kiesel, "Sterben in der Schönen Literatur », in Thomas Fuchs (éd.), Menschenbild und Menschenwürde am Ende des Lebens, Winter, Heidelberg 2010, pp. 199-210.

Katharina Kofmehl-Heri, Die Seniorenbühne : ein neues Stück populärer Theaterkultur in der Schweiz, Institut für Populäre Kulturen der Universität Zürich, Zürich 2006.

Thomas Küpper, Das inszenierte Alter : Seniorität als literarisches Programm von 1750 bis 1850, Königshausen \& Neumann, Würzburg 2004.

Joern Rauser, „Über die Herbstwelten in der Literatur“ : Alter und Altern als Themenkomplex bei Hans Henny Jahnn und Arno Schmidt, Peter Lang, Frankfurt am Main 2001.

Pat Thane (éd.), Das Alter. Eine Kulturgeschichte, trad. Dirk Oetzmann et Horst M. Langer, Primus Verlag, Darmstadt 2005.

\section{NOTES}

1.

Cf. Pat Thane (éd.), Das Alter. Eine Kulturgeschichte, trad. Dirk Oetzmann et Horst M. Langer, Primus Verlag, Darmstadt 2005, pp.54-62; Joern Rauser, „Über die Herbstwelten in der Literatur“: Alter und Altern als Themenkomplex bei Hans Henny Jahnn und Arno Schmidt, Peter Lang, Frankfurt am Main 2001; Helmuth Kiesel, « Das Alter in der Literatur », in Ursula Staudinger (éd.), Was ist Alter(n)? Neue Antworten auf eine scheinbar einfache Frage, Springer, Heidelberg 2008, pp.173-188 et "Sterben in der Schönen Literatur », in Thomas Fuchs, Menschenbild und Menschenwürde am Ende des Lebens, Winter, Heidelberg 2010, pp.199-210; Thomas Küpper, Das inszenierte Alter: Seniorität als literarisches Programm von 1750 bis 1850 , Königshausen \& Neumann, Würzburg 2004. 
2. Pour leur aide précieuse, je remercie les responsables des maisons d'éditions théâtrales, en particulier Thomas Maagh (Verlag der Autoren) et Brigitte Falk (Gustav Kiepenheuer), les artistes Philipp Löhle et Stefan Kaegi ainsi que les services dramaturgiques au Theater Baden-Baden et au Badisches Staatstheater Karlsruhe.

3. Cf. Andrea Gröppel-Klein, „Die „schizophrene Beziehung“ von Marken zum Alter“, in Andreas Kruse (éd.), Leben im Alter. Eigen- und Mitverantwortlichkeit in Gesellschaft, Kultur und Politik, AKA Verlag, Heidelberg 2010, pp. 117-132.

4. „Altern ist ein lebenslanger Prozeß, der mit der Geburt beginnt und mit dem Tode endet. [...] Die Abgrenzung eines eigenen Lebensabschnitts ,Alter' ist im Grunde nicht möglich.“ Andreas Kruse et Hans-Werner Wahl (éd.), Zukunft Altern. Individuelle und gesellschaftliche Weichenstellungen, Spektrum, Heidelberg 2010, pp. 7-9.

5. Cf. Eva Males, «Deutschland: Europas künftige Rentnerrepublik », in Die Presse, 20 février 2010.

6. „Unsere Gesellschaft befindet sich in einem demografischen Wandel. Wir leben in einer Welt, in der es immer mehr alte Menschen geben wird. [...] Jemand, der sich heute mit 50 Jahren aus der Arbeitswelt zurückzieht, wird unter Umständen 100 Jahre alt. Ein halbes Jahrhundert Freizeit." Archives du Theater Baden-Baden.

7. Katja: Frauen werden im Schnitt fast zehn Jahre älter als Männer. Deshalb bin ich, vom Ende her gesehen, jünger als du. Wenn du noch zwanzig Jahre hast, habe ich noch dreißig. Wenn du noch zehn hast, habe ich immer noch zwanzig und wenn du tot bist, muss ich noch zehn Jahre rumbringen. /Karl: Aber deshalb bin ich doch nicht alt. Warum bin ich denn plötzlich alt, nur weil ich nicht mehr arbeite? Philipp Löhle, Morgen ist auch noch ein Tag, Verlag Autorenagentur, Berlin 2008, p. 49.

8. Lore: Es ist ganz einfach. Du stellst dich an eine Vorfahrtsstraße oder einen Zebrastreifen - die besten Zeiten sind, früh zwischen sieben und neun und abends zwischen fünf und sieben - und wartest bis einer mit seinem Auto vorbeikommt. Wenn er ungefähr noch zehn, fünfzehn Meter entfernt ist, springst du auf die Straße oder den Zebrastreifen und ihm vors Auto. [...] Bestenfalls brichst du dir vielleicht einen Arm oder verstauchst dir einen Knöchel. [...] Der junge Kerl ist erst mal den Führerschein los und seine Versicherung stockt deine Rente auf. Ibidem, p. 43-44.

9. Nis-Momme Stockmann, Der Mann der die Welt aß, schaefersphilippen, Köln 2009.

10. Lisa: Es geht dem nicht gut ohne deine Mutter. [...] Der hat den Kindern von der Zunge erzählt. Und von seinen Brandblasen. [...] Dem geht's nicht gut./Sohn (stöhnt) : Ja, der ist erkältet./Lisa: Ich glaube... Also langsam musst du dir mal Gedanken machen... Ich glaub, er braucht jemanden./Sohn : Quatsch. So schlimm ist das nicht./Lisa: Der wird alt. Und wirr im Kopf. Der kriegt das alleine alles nicht mehr klar./Sohn: Der hat nur ne kleine Grippe./Lisa: Und dass der sich die Zunge abbeißt? Wo der doch nur noch so schlechte Zähne hat. [...] Der braucht Betreuung./Sohn. Der ist doch erst 64./Lisa : 67./Sohn: Der arbeitet doch noch!/Lisa: Ja, gerade deshalb. Wenn er da nen Fehler macht... Der ist doch auch gar nicht versichert und nix. (Ibidem, p.10.)

11. Une image pessimiste de la vieillesse se trouve dans Fin de partie où les vieux parents sont mis à la poubelle. Thomas Bernhard a été rapproché de cette dramaturgie de la noirceur. Par ailleurs, la figure grand-paternelle est récurrente dans les œuvres de Thomas Bernhard comme l'affirment les articles réunis par Franz Gebesmair et Manfred Mittermayer (Bernhard-Tage Ohlsdorf 1999. "In entgegengesetzter Richtung»: Thomas Bernhard und sein Großvater Johannes Freumbichler, Bibliothek der Provinz, Weitra 2000).

12. Thomas Bernhard, Minetti: ein Portrait des Künstlers als alter Mann, Suhrkamp, Frankfurt am Main 1978.

13. Cf. Anne Deshoulières, «Le théâtre du "vieillir" dans La ménagerie de verre et Un tramway nommé désir de Tennessee Williams ", in Alain Montandon (éd.), Figures du vieillir, Presses Universitaires Blaise Pascal, Clermont-Ferrand 2005, p. 173-193. 
14. IRIS : Redet ihr nicht mehr über eure Gesichter?/MARTHA : Wir wollen unsere Gesichter nicht mehr sehen. [...] Ich verstehe jeden, der mich vor zwanzig Jahren gekannt hat und mich jetzt nicht mehr anschauen will. [...] In meiner Jugend war ich weiß wie Porzellan und zart wie ein Engel. Robert hat mich angeschaut und hat gedacht: Mein Gott, die schaut aus wie ein Glas Milch. [...] Wie ein Glas Milch habe ich mich gefühlt. Irgendwann nimmt mich einer an die Lippen und schluckt mich weg. Dann bin ich gerettet. (Wilhelm Genazino, Lieber Gott, mach mich blind/Der Hausschrat, Hanser, München 2006, pp. 10-11).

15. MARTHA : Wie unvorstellbar zufrieden war ich einmal mit meinen Haaren! Als ich neun war, sah ich in den Spiegel und war sofort glücklich. Ich habe mich fast ununterbrochen gekämmt. Dabei fielen mir schon als Kind die Haare aus. Aber damals war Haarausfall etwas Wunderbares. Damals wuchsen meine Haare schnell nach. [...] Jetzt schimmert meine Kopfhaut immer mehr durch. Ich ekele mich vor dem Rosa meines blank werdenden Kopfes. Die Farbe Rosa bei älteren Frauen bedeutet: Vorsicht, ich werde euch ekeln! Ach, welche Hoffnungen habe ich einmal in meine Haare gesetzt. (Ibidem, p. 54)

16. ROBERT : [...] Schau, als wir uns liebten, waren wir gerade noch jung gewesen. Es genügte, daß wir uns nebeneinander legten, es genügte, daß wir an einem Tisch saßen. Das ist lange her. Heute können wir noch so lange nebeneinander liegen, es regt sich nichts mehr, weder bei dir noch bei mir. [...] Wenn zwei Leute älter geworden sind und die Liebe nicht mehr klappt, gibt es zwei Möglichkeiten. Die erste ist, das Paar ist klug und hält die Ermattung für natürlich und stellt den Betrieb ein. Die zweite ist, das Paar erfindet sich eine Perversion, die eine Fortsetzung der Sexualität erlaubt, mit anderen Mitteln sozusagen. (Ibidem, pp. 36-37)

17. ELSE : Nehmen Sie Potenzpillen? /KARL : Nur im Notfall./ELSE : Also immer. Funktionieren die Pillen bei Ihnen ? / KARL : Mehr oder weniger./ELSE : Also weniger. Muß die Frau bei Ihnen... äh... handgreiflich werden? /KARL : Mehr oder weniger./ELSE : Also mehr. (Der Hausschrat, p.117)

18. KARL: Sie macht Fortschritte./BRUDER : Sie ist gelähmt. Sie kann nicht sprechen./KARL : Ich kann sie pflegen./BRUDER: Du bist auch nicht der Jüngste./KARL: Ich schaff das./BRUDER: Du übernimmst dich./KARL: Das ist kein Problem./BRUDER : Das ist egoistisch./KARL: Das sagst du./ BRUDER: Und wenn Du ein Heim suchst? /KARL : Nein./BRUDER: Warum nicht? /KARL: Sie will in kein Heim. (Jan Neumann, Liebesruh, Gustav Kiepenheuer Verlag, Berlin 2005, p. 32).

19. KARL : Du sollst in ein Pflegeheim./REGINE : Nein./KARL : Ich habe Tabletten./KARL : Da schläfst du ein und wachst nicht mehr auf./REGINE: Ja. [...] Sie schläft ein/KARL: Ich habe Angst. /Er erwürgt sie. (Ibidem, pp. 40-42).

20. Ob er den unterschied noch merkt/zwischen dem medizinkram da/und sagen wir/ner leberwurststulle/der frisst das eine wies andre/ ohne zu denken/und ohne appetit/nur weil's ihm einer sagt /friss jetzt ede/schon wirft er sich's rein/sei mal ehrlich/willste dein leben/in dem zustand/auch so verlängert wissen/so mit gewalt im dämmern/zwischen leben und tod/ zurückgezerrt zu werden immer wieder/wo doch der tod erleichterung wär (Ralf-G. Krolkiewicz, sonst is alles wie immer, Drei Masken Verlag, München 2003, p. 33).

21. BERTA Lerne ich einen Mann kennen, sage ich ihm nie, was ich für einen Beruf habe. [...] Meistens sage ich, ich heiße Maria und habe ein Nagelstudio. [...]/CARLA Klingt auf jeden Fall besser als, ich bin Berta, die Altenpflegerin./ BERTA Oder ich bin Carla, die Seniorendompteuse./ CARLA Oder Berta, die Greisenputze. (Theresia Walser, King Kongs Töchter, Verlag der Autoren, Frankfurt am Main 1999, pp. 11-12).

22. L'envie de jouer les anges de la mort structure la comédie policière Arsenic and old lace (Arsenic et vieilles dentelles) écrite par Joseph Kesselring (New dramatists play service, New York [1941]). Créée en août 1941 à Broadway, cette comédie a été rendue célèbre par le film de Frank Capra qui est sorti en salle en 1944.

23. MEGGIE [...] Und wenn hier was abkratzt, was aussieht wie Ötzi, dann gehe ich aber nachher, wenns sein muß, mit dem Lötkolben dran, dann sieht das immerhin noch aus als hätte Ötzi 
vorher noch die Sophia Loren gevögelt. [...] Doch, doch, da muß man übertreiben, da gabs ganz andere Zeiten, da hat man denen Trauben in die Haare geflochten, den Mund voll mit Weizen und tschüss. (King Kongs Töchter, p. 39).

24. B ERTA Brav, altes Mädchen, brav, dich mach ich heute Nacht zum großen Kitsch, zur Sexgöttin aus der Mottenkiste, in deinen Untergang leg ich mein ganzes Herzblut./MEGGIE Mal ihr den Mund nicht zu groß, Berta./ BERTA 80 werden, was altes Mäuschen, 80 werden [...] wer will schon zusehen, wie du hier langsam in die Matraze sickerst. (King Kongs Töchter, p. 45)

25. Karl Wittlinger, Gesegnete Mahlzeit (Hochdeutsche Fassung), Felix Bloch Erben, Berlin [1991]. Cette comédie existe aussi dans deux versions dialectales. Le titre de la version alémanique, Tyrannenmord im Altenheim, est bien plus explicite que le titre hanséatique Pension Sünneschien (créée au Ohnsorg Theater de Hambourg en 1991).

26. Elfie (unterbricht) Na und - : Wenn die Jahre kommen, wo wir Mädchen nicht mehr kriejen wat wir brauchen, und die Knaben nich mehr so recht können wat se wollen - da stellt sich leicht der sojenannte « Altersfrust » ein. Schuld daran sind die Hormone-: hat ein berühmter Forscher spitzjekriegt in sein Labor. Und festjestellt__ Dajejen is der Porno de anjemessne Medizin. (Ibidem, p. 54).

27. Markus Köbeli, Zimmer frei, Gustav Kiepenheuer Verlag, Berlin 1990. Depuis sa création au Stadttheater Konstanz en février 1991, la pièce a été montée par 35 théâtres en Allemagne et en Suisse jusqu'en 2011.

28. (Schweigen, Opa schaut zum Sarg hinüber.)/GLOTZ: Meine neueste Anschaffung. Schick, nicht ? /OPA : Ganz apart, ja./GLOTZ : Was heißt da apart. Absolut smashing ist der. Wissen Sie, was so ein Ding kostet? Dafür läßt man ganz schön Kohle./OPA : Ich weiß. Ich habe mich auch schon mal erkundigt./GLOTZ : Warum ? Stehen Sie auch auf Särge ? /OPA : Neinein. Mein Interesse ist rein altersbedingt. Ich bin für geregelte Verhältnisse, verstehen Sie? / GLOTZ : Und ich mehr fürs Kaos. Leben, aber subito. Darum habe ich auch diesen Sarg gekauft. (Ibidem, p. 6).

29. Leo kommt herein, auf dem Tisch liegen ein frisches Handtuch und eine kleine Schale mit Tabletten. Leo hängt sich das Handtuch um, schüttet sich die Tabletten in die Hand, geht zum Fenster, öffnet es, sieht sich um, dann schmeißt er die Tabletten in hohem Bogen nach draußen, er schließt das Fenster. (Lutz Hübner, Das Herz eines Boxers, Klett, Stuttgart 2009, p. 4 [Hartmann \& Stauffacher Verlag, Köln 1996].

30. Die Pantoffeln und die Strickweste bleiben sauber, du wirst gleich hübsch in Folie verpackt, ich hab sogar durchsichtige mitgebracht, damit du mir schwer beeindruckt beim Streichen zusehen kannst. Kommt ja schließlich nicht alle Tage vor, dass hier im Heim einer arbeitet, was? Klar, Strohsterne und so'n Tinnef ist ja auch'ne Schweinearbeit. [...] Was glotzt du denn so ? Der erste Besuch seit dem Krieg, was? Glaub bloß nicht, dass ich das hier aus Menschenfreude mache. Seh ich aus wie jemand, der ein Herz für Senioren hat ? Ibidem.

31. Le langage simplifié, utilisé par certains soignants, infantilise les personnes âgées, sousentendant que la personne âgée est dépendante et nécessite de l'aide extérieure. L'emploi de ce langage, vécu comme méprisant, car réfutant l'autonomie potentielle, peut renforcer le sentiment de dépendance. Cf. Ulla Walter et Hartmut Remmers, „Altersbilder in Medizin und Pflege“, in Andreas Kruse (éd.), Leben im Alter. Eigen- und Mitverantwortlichkeit in Gesellschaft, Kultur und Politik, AKA Verlag, Heidelberg 2010, pp. 103-115.

32. „Ich war vorher in einem Heim, in dem ich mich richtig wohlgefühlt habe. [...] da hab ich mir einmal in die Hose gemacht, und das war mir sehr peinlich. Weißt du, es ist nicht immer einfach, alt zu sein, wenn einen die Leute wie einen Idioten behandeln. Und da war dieser Pfleger, ich hab ihn nie leiden können, so einer wie du, als du vorgestern hier reingekommen bist, so einer, der mit dir umgeht, als wäre man ein Meerschweinchen. Er holt die Wäsche ab und bemerkt mein Missgeschick. Da brüllt er, so dass alle es hören können: He, Leo, wird wohl langsam Zeit für die Windeln. Da hab ich mich sehr geschämt, aber ich dachte: Du bist nur ein kleiner Rotzlöffel, du wirst auch noch alt, schneller als du denkst. Aber als er dann am nächsten Tag kommt und mich 
mit so einem gemeinen Lächeln fragt, ob ich denn noch trocken sei, ist mir die Wut gekommen. Weißt du, ich war einmal berühmt für meine Linke, den Stahlhammer haben sie mich genannt. Er ging sofort zu Boden [...]“" (Das Herz eines Boxers, p. 16).

33. Ibidem, p. 23. Revenant sur les diverses mises en scène de sa pièce, Lutz Hübner souligne que les acteurs portent la pièce qui se nourrit de la qualité de leur rencontre. „Große Konzepte muss eine Regie da nicht entwickeln, was es braucht, ist eine gute Schauspielerführung und ein einfaches praktikables Bühnenbild. [...] Oft spielen der dienstälteste Spieler des Ensembles und der Schauspielanfänger das Stück und da entwickelt sich meist eine Freundschaft, die der des Stückes ähnelt. Daraus kommt die Energie der Inszenierung." (Ibidem, p. 62).

34. "Abends beim Schreiben von "Josef und Maria" denke ich mir, daß mein Stück gut ausgehen soll. Dies soll mein erstes Stück werden, das gut ausgeht. Unbedingt. Ich habe keinen Bedarf mehr an Katastrophen.» (Brief an einen Freund, 1980, cité in Peter Turrini, Josef und Maria (Neufassung 1999), Suhrkamp, Frankfurt am Main 2003 [Frischfleisch \& Löwenmaul, Wien 1980], p. 61. La pièce a été créée en 1980 au Volkstheater à Vienne.

35. „Das Bild, welches die Gesellschaft von alten Menschen hat, wird am wenigsten von den Alten selber bestimmt. Die Werbung entdeckt sie als „spezifische Käuferschicht“, das Fernsehen macht sie zu fröhlichen „Senioren“, und selbst die wissenschaftliche Soziologie findet an den alten Menschen das, was sie im vorhinein annimmt: dass sie eine unterprivilegierte Minderheit sind. Ich wollte kein Stück „über“ alte Menschen schreiben. Ich wollte ihre Geschichten, ihre Erzählungen, ihre Erinnerungen aufnehmen und annehmen. Meine Aufgabe als Dramatiker bestand darin, diese Geschichten auszuwählen, zu ordnen und zwei alte Menschen in eine dramatische Situation zu bringen (Text für ein Programmheft, 1980, cit. in ibidem, p. 58).

36. MARIA liest „Ich möchte mein Herz ausschütten, helfen kann mir ja doch niemand. Meine Schwiegertochter verschließt vor mir die Lebensmittel, ich darf nichts nehmen und bin immer hungrig. Meine Freundinnen zahlen mir ab und zu einen Kaffee. Die Rente kassiert die Schwiegertochter, sie gibt nichts davon her. Ich bin siebenundachtzig Jahre alt. Habe ich das auf meine alten Tage verdient? Hochachtungsvoll.“ (Peter Turrini, Josef und Maria (Neufassung 1999), op. cit., pp. 18-19).

37. Wissen Sie, Herr Josef Pribil, daß ich vierzig Jahre so getan habe, als wäre ich nie beim Varieté gewesen? Oft ist es mir schwer angekommen, beim Kapsch, der Radiofirma, wenn ich den Akkord überzogen hab [...] Und vor ein paar Tagen, wie meine Schwiegertochter hinter meinem Sohn steht und er sagt : Mama, bitte komme nicht am Heiligen Abend, es gibt sonst nur Unfrieden, da hab ich in ihr freches und grausliches Gesicht geschaut und mir gedacht, du warst nie beim Varieté. Du warst nie beim Varieté! [...] Ihr könnts eine alte Frau umbringen, aber eine Artistin geht nicht unter! (p. 24)

38. Josef schaut sie an. Schweigen. Josef bewegt seine Hand unter der Decke./JOSEF Du hast aber feine Haut, Maria./Schweigen./MARIA Ach, Josef, vor vierzig Jahren hättest du mich kennenlernen sollen./JosEF Da war ich ein verhungerter Arbeitsloser, lungenkrank, dauernd am Kommissariat. So etwas hättest du bestimmt nicht angeschaut, Maria, es hat mich ja keine Frau angeschaut, dazumal. [...]/MARIA Bist du kitzelig, Josef ? (Ibidem, p. 49).

39. Cf. Peter Turrini, Josef und Maria, op. cit., p. 58.

40. Katharina Kofmehl-Heri, Die Seniorenbühne: Ein neues Stück populärer Theaterkultur in der Schweiz, Institut für Populäre Kulturen der Universität Zürich, Zürich 2006 [Zürcher Beiträge zur Alltagskultur, Band 16], p. 341.

41. „Weibliche Lebenserfahrung und geschlechtsspezifisch geprägte Lebensläufe bestimmen die Alltagsgestaltung im Alter weiterhin. [...] Als persönliche Gründe fürs Theaterspielen nannten die Frauen den „Wunsch, als Frau auch einmal im Rampenlicht zu stehen“, „im Rollenspiel geheime Wünsche auszuleben“ oder „eine Bestätigung der eigenen Erfahrungen zu erfahren“. Diese Aussagen verweisen auf die Lebenserfahrungen älterer Frauen in unserer Gesellschaft: Als Ehefrauen unterstanden sie rechtlich dem Ehemann, in der Arbeitswelt besetzten sie vorwiegend 
die untergeordneten Stellen, auf Grund des Geschlechts verdienten sie prinzipiell weniger als Männer, und durch die späte Einführung des Frauenwahlrechts waren sie lange politisch unmündig. Hinzu kommt, dass ihre weibliche Sozialisation sie früher daran hinderte, einen Platz in der Öffentlichkeit einzunehmen." (Ibidem, p. 344).

42. Rimini Protokoll a mis ses archives en ligne (http://www.rimini-protokoll.de).

43. Frau Döring mit der Schleuder. Sie sagt: Früher dachte ich, älter wird man erst viel später. Frau Simon sagt, die höchste Geschwindigkeit ist, wenn ich hinfalle und mir einen Oberschenkelhals breche. Das können sie nicht darstellen. (retranscription, archives vidéo, http://www.rimini-protokoll.de/website/de/audio_video_date.html)

44. On trouve une galerie des photos du spectacle sur le site http://www.pvc-tanz.de/ $\sim$ programm/projekte/78.

45. Les archives en ligne du collectif (http://www.sheshepop.de/produktionen/testament/\#c194) renvoient notamment à un reportage de l'émission culturelle aspekte sur le projet (diffusé sur la deuxième de la télévision publique allemande).

\section{RÉSUMÉS}

Le théâtre contemporain germanophone propose une grande diversité des images de la vieillesse qui reste une période de vie difficilement définissable dans une société qui vieillit mais prône activisme permanent et jeunesse éternelle. Les auteurs comme Philipp Löhle et Nis-Momme Stockmann font écho aux changements démographiques et éclairent la difficulté d'accepter la retraite ou la démence. Les sujets effrayants comme le délabrement physique, la maladie, la perte de l'autonomie, la solitude ou la mort sont souvent abordés par le grotesque. Chez Wilhelm Genazino, la nostalgie obsède les personnages qui refusent de vieillir. À l'opposé des pièces pessimistes, des auteurs comme Peter Turrini, Markus Köbeli et Lutz Hübner décrivent l'épanouissement des personnes âgées ou des cohabitations intergénérationnelles réussies. L'écriture théâtrale par le plateau, notamment les projets scéniques de Rimini Protokoll, et le théâtre amateur des seniors valorisent, par un réalisme bienveillant, les témoignages des personnes âgées.

Contemporary German-language plays offer quite diverse images of old age, a life period which is hard to determine in a society becoming older and older but promoting permanent activism and eternal youth. Treating the demographic change, dramatists such as Philipp Löhle and NisMomme Stockmann examine the difficulty in accepting retirement or dementia. Quite often grotesque images are used in order to approach appalling topics such as physical decline, disease, loss of autonomy, solitude or death. Refusing to grow old, the figures in the theatre of Wilhelm Genazino are obsessed by nostalgia. In opposition to the wide range of pessimistic plays, authors like Peter Turrini, Markus Köbeli and Lutz Hübner show us how old people can attain fulfilling lives and start living together successfully with members of other generations. New scenic forms, especially the artistic projects of Rimini Protokoll, and the amateur theatre for seniors, using a generous realism, give more value to testimonies of old people.

Das zeitgenössische deutschsprachige Theater zeigt vielfältige Bilder des Alters, das jedoch ein nur schwerlich definierbarer Lebensabschnitt in einer alternden Gesellschaft ist, die zugleich stetiges Aktivsein und ewige Jugend einfordert. Autoren wie Philipp Löhle und Nis-Momme 
Stockmann gehen auf die demografischen Veränderungen ein und beleuchten die Schwierigkeit, den Rückzug aus dem Berufsleben oder Demenz zu akzeptieren. Oft werden über groteske Bilder die mit Angst besetzten Themen wie körperlicher Verfall, Krankheit, Verlust der Selbstständigkeit, Einsamkeit oder Tod angesprochen. Bei Wilhelm Genazino weigern sich die besessen nostalgischen Figuren zu altern. Im Gegensatz $\mathrm{zu}$ den zahlreichen pessimistischen Texten beschreiben Autoren wie Peter Turrini, Makus Köbeli und Lutz Hübner, wie alte Menschen ein erfülltes Leben finden und mit Menschen der anderen Generationen erfolgreich zusammenleben können. Neue Formen des szenischen Schreibens, insbesondere die Bühnenprojekte von Rimini Protokoll, sowie das Seniorentheater geben durch wohlwollenden Realismus den Zeugenberichten alter Menschen einen neuen Stellenwert.

\section{INDEX}

oeuvrecitee Das Herz eines Boxers, Der Hausschrat, Der Mann der die Welt aß, Die Bremer Stadtmusikanten, Ein Fest für Boris, Gesegnete Mahlzeit, Josef und Maria, King Kongs Töchter, Lieber Gott mach mich blind, Liebesruh, Minetti, Morgen ist auch noch ein Tag, Mütter.Väter.Kinder, sonst is alles wie immer, Theresia Walser, Zimmer frei Mots-clés : Allemagne, théâtre contemporain, vieillesse

\section{AUTEURS}

\section{ANDREAS HÄCKER}

Université de Strasbourg 